\title{
Intestinal Microbiome Shifts, Dysbiosis, Inflammation, and Non-alcoholic Fatty Liver Disease
}

\author{
Emma T. Saltzman ${ }^{1,2 \star}$, Talia Palacios ${ }^{1,2}$, Michael Thomsen $^{1,2}$ and Luis Vitetta ${ }^{1,2 *}$ \\ ${ }^{1}$ Sydney Medical School, University of Sydney, Sydney, NSW, Australia, ${ }^{2}$ Medlab Clinical, Sydney, NSW, Australia
}

\section{OPEN ACCESS}

Edited by:

Gayane Manukyan,

Institute of Molecular Biology

(NAS RA), Armenia

Reviewed by:

Michael Kogut,

Agricultural Research Service (USDA),

United States

Mario M. D'Elios,

University of Florence, Italy

${ }^{*}$ Correspondence:

Emma T. Saltzman

esal4025@uni.sydney.edu.au

Luis Vitetta

Iuis.vitetta@sydney.edu.au; luis_vitetta@medlab.co

Specialty section

This article was submitted to Microbial Immunology,

a section of the journal

Frontiers in Microbiology

Received: 02 August 2017 Accepted: 10 January 2018 Published: 30 January 2018

Citation:

Saltzman ET, Palacios T, Thomsen M and Vitetta L (2018) Intestinal Microbiome Shifts, Dysbiosis,

Inflammation, and Non-alcoholic Fatty Liver Disease. Front. Microbiol. 9:61. doi: 10.3389/fmicb.2018.00061
Adverse fluctuations in the distribution of the intestinal microbiome cohort has been associated with the onset of intra- and extra-intestinal inflammatory conditions, like the metabolic syndrome (MetS) and it's hepatic manifestation, non-alcoholic fatty liver disease (NAFLD). The intestinal microbial community of obese compared to lean subjects has been shown to undergo configurational shifts in various genera, including but not limited to increased abundances of Prevotella, Escherichia, Peptoniphilus, and Parabacteroides and decreased levels of Bifidobacteria, Roseburia, and Eubacteria genera. At the phylum level, decreased Bacteroidetes and increased Firmicutes have been reported. The intestinal microbiota therefore presents an important target for designing novel therapeutic modalities that target extra-intestinal inflammatory disorders, such as NAFLD. This review hypothesizes that disruption of the intestinal-mucosal macrophage interface is a key factor in intestinal-liver axis disturbances. Intestinal immune responses implicated in the manifestation, maintenance and progression of NAFLD provide insights into the dialogue between the intestinal microbiome, the epithelia and mucosal immunity. The pro-inflammatory activity and immune imbalances implicated in NAFLD pathophysiology are reported to stem from dysbiosis of the intestinal epithelia which can serve as a source of hepatoxic effects. We posit that the hepatotoxic consequences of intestinal dysbiosis are compounded through intestinal microbiota-mediated inflammation of the local mucosa that encourages mucosal immune dysfunction, thus contributing important plausible insight in NAFLD pathogenesis. The administration of probiotics and prebiotics as a cure-all remedy for all chronic diseases is not advocated, instead, the incorporation of evidence based probiotic/prebiotic formulations as adjunctive modalities may enhance lifestyle modification management strategies for the amelioration of NAFLD.

Keywords: intestinal microbiome, intestinal epithelial cell dysbiosis, dysbiosis, macrophage, inflammation, mucosal immunity, NAFLD

\section{INTRODUCTION}

NAFLD is a growing public health concern, laying claim to both a steadily rising prevalence as well as an increasingly young age at diagnosis (Welsh et al., 2013; Nobili et al., 2014). These trends reflect the increased rates of risk factors associated with an obesogenic lifestyle and the development of type 2 diabetes mellitus (T2DM) (Targher et al., 2007; Welsh et al., 2013). The prevalence of NAFLD in obese adults or those with T2DM has been reported to be 67.5 and $74 \%$, respectively, 
(Williams et al., 2011; Paquissi, 2016) compared to only $25 \%$ in the general adult population (Younossi et al., 2016). As a spectrum of diseases, NAFLD has been associated with significant morbidity and mortality, with advanced forms of the disease expressed as fibrosis, cirrhosis, and hepatocellular carcinoma (HCC).Additionally, insulin resistance (IR) and obesity have been identified as NAFLD risk factors (Gaggini et al., 2013), with NAFLD also reported to increase the risk of T2DM and cardiovascular disease, justly classifying NAFLD as the hepatic manifestation of the metabolic syndrome (MetS) (Adams et al., 2005; Targher et al., 2007, 2010; Dunn et al., 2008; Starley et al., 2010; Gregor and Hotamisligil, 2011; Lumeng and Saltiel, 2011; Dietrich and Hellerbrand, 2014; Paolella et al., 2014; Paquissi, 2016). NAFLD hence presents as a multi-systemic disease (Starley et al., 2010; Paolella et al., 2014). In light of the potential of NAFLD to progress to an ever-increasing prevalence, an appreciation of the molecular mechanisms that facilitate the manifestation of NAFLD disease chronicity and the pathways that trigger the transition across the spectrum of diseases is a pertinent aspect in designing novel effective therapeutic modalities. With the purported pathogenic mechanisms of NAFLD being intertwined with peripheral IR, increased liver lipolysis is reported to contribute to increased levels of hepatic free fatty acids (FFAs) (Paolella et al., 2014). IR simultaneously triggers increased gluconeogenesis and reduced glyconeogenesis, which further increases the production of circulating FFAs (Paolella et al., 2014).

The definition of NAFLD covers a spectrum of liver histologies, ranging from the benign and usually non-progressive simple steatosis (SS) (Tilg and Moschen, 2010; characterized by the accumulation of lipid droplets that exceed $5 \%$ of the total weight of the liver) to non-alcoholic steatohepatitis (NASH) which is found in $30 \%$ of NAFLD patients and is described as the beginning stages of inflammation and lobular ballooning which results from persistent hepatic injury (Jiang et al., 2015). Chronic inflammation can cause the liver to respond with compensatory tissue repair, a mechanism that initiates fibrosis and even cirrhosis through collagen deposition and scarring-which eventually forms the foundation of hepatocellular cancer (HCC) in extremely rare cases (Tilg and Moschen, 2010; Bieghs and Trautwein, 2014). Systems biology continues to probe the pathophysiology of this disease and the factors that establish it's manifestation and drives disease progression across the spectrum toward severe phenotypes.

The manifestation and progression of NAFLD is tentatively attributed to a range of factors as part of the multiple parallel hits hypothesis, which postulates that NAFLD is the result of inflammation in the liver induced by numerous intestinal-derived or adipose tissue-derived triggers (Tilg and Moschen, 2010). With the first hit said to be the onset and maintenance of SS, and the additional hits of gut-derived endotoxins and pro-inflammatory cytokines from adipose tissue reported to provide the impetus for NASH development and subsequent progression (Day and James, 1998). This hypothesis is flagging the importance of aberrant innate immunity as a central pathway for NAFLD progression (Miele et al., 2009;
Tilg and Moschen, 2010) along with stress signaling networks and circulating adipocytokines and pro-inflammatory cytokines (Miele et al., 2009; Tilg and Moschen, 2010).

Although the molecular pathways that lead to the pathogenesis and progression of NAFLD remain poorly understood, it is accepted that inflammation is a major factor in hepatic injury (Bieghs and Trautwein, 2014). Currently, experimental data suggests that interactions of the innate immune system with the different resident liver cell types help perpetuate and maintain adverse inflammatory responses in the liver (Starley et al., 2010; Farrell et al., 2012; Bieghs and Trautwein, 2014). Serum markers of inflammation, including C-reactive protein (CRP), interleukins (ILs), and other general immunity markers are associated with the diagnosis and prognosis of NAFLD (Chiang et al., 2010; Harley et al., 2014), whilst at the cellular level, data has implicated an imbalance in $\mathrm{T}$ helper 17 (Th17) cells over regulatory $\mathrm{T}$ (Treg) cells, that occurs from an over-differentiation of T helper cells (Hammerich et al., 2011). A disturbance to the equilibrium between Th17 and Treg cells is a key event in the initiation of pro-inflammatory activity.

The innate immune system responds to cell damage or pathogenic invasion through pattern recognition receptors (PRRs), that are expressed intracellularly or on the surface of resident liver cells (Bieghs and Trautwein, 2014). These PRRs are programmed to detect damage-associated molecular patterns (DAMPs) that are released by injured cells or pathogen-associated molecular patterns (PAMPs), which are derived from intestinal bacterial metabolites (Pedra et al., 2009; Takeuchi and Akira, 2010). From a consideration of the consequences of intestinal epithelial dysbiosis, this review hypothesizes that the cascade of signals that activate adverse innate immune system and inflammatory activity implicated in NAFLD pathophysiology are triggered by the continuous release of endotoxins and other intestinal bacterial-derived products which can reach the liver through the gut-liver axis interface.

Resident liver cells have their own PRRs in toll-like receptors (TLRs). Activating these TLRs is an important step in the development of NAFLD as they are responsible for inducing gene transcription that facilitates responses of the innate immune system (Takeuchi and Akira, 2010). Kupffer cells, stellate cells, and hepatocytes amongst others, express TLRs and recognize a large array of PAMPs, which enable pro-inflammatory activity by activating different liver cells. PAMPs and DAMPs as well as irritants from the host's environment are themselves recognized by inflammasomes, receptors of the innate immune system. In response to sensing infectious microbes, inflammasomes are responsible for the pro-inflammatory activity observed in the initiation or manifestation of inflammatory diseases, like NAFLD. NLRP3 is an inflammasome that has been reported to be specifically and critically involved in NAFLD progression, with experimental and clinical data identifying higher levels of expression in NASH-affected subjects.

The intestinal microbiome is central to the narrative that NAFLD manifestation is largely a consequence of dysregulated innate immunity in response to persistent pro-inflammatory activity. The role of the intestinal microbiome is multi-factorial, functioning as an immunological, metabolic, and protective 
tool for optimal host health. When the intestinal microbiome is in dysbiosis, the health of the host is compromised as the microbiome is unable to maintain control of local homeostasis, increasing intestinal permeability. Disruption to intestinal epithelial homeostasis leads to hepatic exposure to exogenous and endogenous antigens that drives hepatoxic influences via the gut-liver axis interface (Glavan et al., 2016).

\section{THE INTESTINAL MICROBIOME AND THE EPITHELIAL BARRIER}

The microbial ecosystem of the gastrointestinal tract (GIT) comprises a metabolically and immunologically complex and active organ (Vitetta et al., 2013). Recognized as the most biodiverse and dense microbial site, the intestinal microbiome is estimated to harbor over $10^{14}$ bacterial cells (Jiang et al., 2015). Serving as a key influence of host health, the GIT is the site that facilitates the exposure of environmental, dietary, and microbial antigens to the immune system (Vitetta et al., 2013). Existing in a state of symbiotic homeostasis, the intestinal microbiome and the immune system largely co-develop from birth (Nicholson et al., 2012). Subject to an array of complex interactions, dependent on host genetics, lifestyle, dietary and environmental cues, the microbiome and host share a bi-directional relationship, with both parties helping to shape each other's development and composition (Nicholson et al., 2012).

The intestinal epithelial barrier is a dynamic network made up of luminal and mucosal components (Paolella et al., 2014); with an epithelial cell layer interlaced with innate mucosal immunity and neuroendocrine elements, encasing the paracellular space that houses the intestinal microbiome diverse niches (Paolella et al., 2014; Ringel et al., 2015). The single layer of epithelial cells that line the intestines is bound by a tight junction protein (TJP) network, serving to form the physical barrier, which provides protection against potential pathogenic assaults and toxins that increases the risk of systemic sepsis. Intestinal epithelial cells have a rapid time of turnover of between 2 and 6 days (Ramachandran et al., 2000). The TJP network also regulates intestinal permeability, providing the pores and channels for passage of molecules (i.e., water, electrolytes, nutrients) by selective permeability (Paolella et al., 2014). The intestinal barrier is also involved in the co-ordination of responses of the innate immune system, with macrophage/dendritic cell activation contributing to host defenses against microbial-induced systemic infections (Paolella et al., 2014). The protrusions that characterize macrophages/dendritic cells allow for the sensation of potential pathogens that have breached the intestinal mucus layers, the mucosa, as well as those sensed in other parts of the intestinal lumen, resulting in the induction of responses such as phagocytosis and of the acquired immune system through B-cell activation (Kumar et al., 2011; Kinnebrew and Pamer, 2012). Figure 1 details the maintenance of the homeostatic state of the intestinal barrier.

Intestinal microbial metabolites provide the substrate for the fermentation of complex dietary carbohydrates to produce short-chain fatty acids (SCFAs), as well as assist the host in harnessing maximal energy from dietary consumption (Jiang et al., 2015). The various metabolites exert varying effects on the host, from the beneficial production of signaling molecules (e.g., butyrate), to inducing mucus and other secretions, to provide the triggers that facilitate the innate mucosal system to maintain local homeostasis. In states of dysbiosis, the intestinal barrier increases in permeability as a result of a disruption to the regulation of the epithelial cell-to-cell tight junction protein network. A compromised intestinal barrier can be associated with bacterial translocation from the gut into the systemic circulation increasing the risk of sepsis. Lipopolysaccharides (LPS), a constituent of gram negative bacteria (Jiang et al., 2015), is found to be increased in the systemic circulation, indicative of dysbiosis (Boulangé et al., 2016). LPS has been associated with inducing apoptosis of lymphocytes under in vivo conditions (Norimatsu et al., 1995; Nielsen et al., 2012; Jiang et al., 2015) demonstrating an immune-modulatory effect. Studies have posited that a loss of lymphocytes in the intestinal mucosa is a consequence of intestinal epithelial dysbiosis and subsequent release of metabolic endotoxins (Jiang et al., 2015). LPS has also been implicated as an inductor of a pro-inflammatory environment which is conducive to MetS, IR and T2DM (Cani et al., 2008). Gram-negative bacteria containing LPS are therefore hypothesized to contribute to NAFLD development. Furthermore, dysbiosis and elevated systemic LPS can be envisaged as markers of intestinal toxicity (Nolan, 2010). Intestinal toxicity driven dysbiosis supports local mucosal inflammatory responses that is concomitant with an increase in intestinal permeability. This combined disruption of the intestinal barrier/mucosal immunity activity can promote and mediate NAFLD pathogenesis via the gut-liver axis (Littman and Pamer, 2011; Wieland et al., 2015).

\section{THE GUT-LIVER AXIS}

The venous system of the portal circulation defines the gut-liver axis and highlights the close anatomical proximity and functional interactions of the gastrointestinal tract and the liver (Paolella et al., 2014; Brandl et al., 2017). The axis is described as a means of enhancing interactions between metabolites of the intestinal microbiome and receptors on the liver, which can trigger a cascade of events that culminates in IR, inflammation of the liver, and eventually the development of liver fibrosis (Paolella et al., 2014). The anatomical and functional link between the gut and liver delivers $70 \%$ of hepatic blood supply via the portal vein. The portal vein is the direct venous outflow from the intestines and thus when the intestinal mucosal barrier is compromised it exposes hepatic tissue to toxic factors derived from the intestines. Therefore, various metabolites produced by intestinal bacteria that reach the liver, have been linked to the manifestation of simple steatosis and NASH (Raman et al., 2013). Dynamic shifts in the gut-liver axis, contributed by either the physical barrier, the microbiome or the liver itself, are a result of alterations to the permeability of the intestinal epithelium and or microbial composition that have been implicated in NAFLD manifestation (Mehal, 2012). Experimental and clinical evidence increasingly implicate dysfunctions of the gut-liver axis in the development 


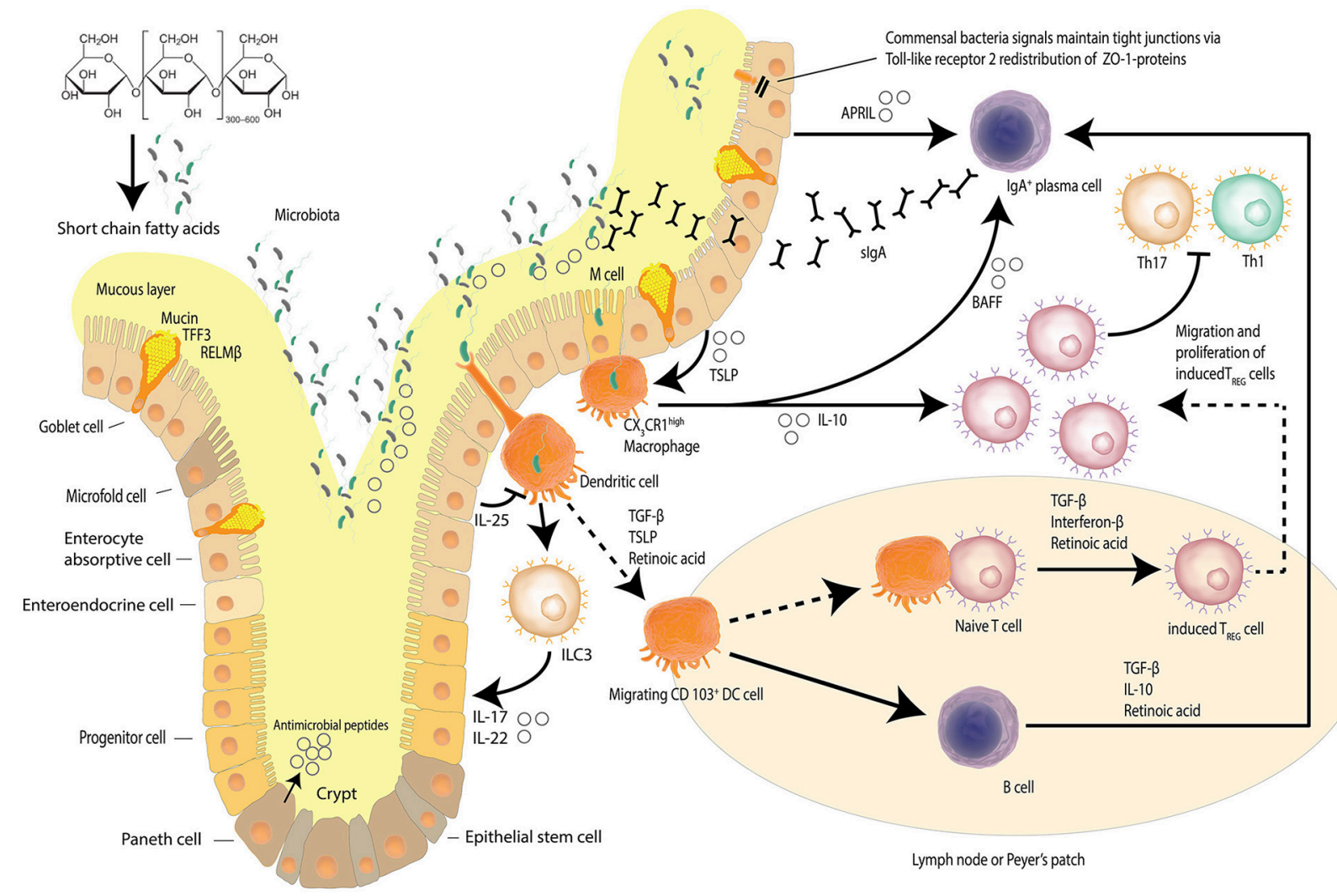

FIGURE 1 | Intestinal epithelial barrier and homeostasis. Several mechanisms, including a mucus layer, antimicrobial peptides and a tight junction protein network collaborate to ensure that the intestinal barrier is not compromised. Goblet cells secrete mucin to provide a protective coating, provide structural integrity and regulate macrophage and adaptive T cell responses during inflammation. Goblet cells also facilitate microbial translocation. The intestinal epithelia network of cells produce a range of soluble protein factors and also express the integrin ligand semaphorin 7A that modulates intestinal $\mathrm{CX}_{3} \mathrm{CR} 1$ macrophage function. Localized CX3CR1 macrophages further release IL-10 to support the proliferation of induced Treg cells and B cell activating factor (BAFF) to further stimulate the production of migrating secretory $\lg A(s \lg A)$ across the epithelium. Dendritic cells sample bacteria and present antigens to other cells including innate lymphoid cells (ILCs). Dietary starch is converted by intestinal bacteria into short chain fatty acids that serve as a source of energy for the epithelial cells but also act as signaling molecules. Signals from commensal bacteria help maintain tight junction via Toll-like receptor 2 (TLR-2) redistribution of ZO-1 proteins. (Kidd, 2003; Wahl, 2007; Hume, 2008; Ogino et al., 2011; Kayama et al., 2012; Abbas et al., 2013; Smith et al., 2013; Zheng, 2013; Ai et al., 2014; Guilliams et al., 2014; Peterson and Artis, 2014; Gottschalk and Kurts, 2015; Robinson et al., 2015; Nakahashi-Oda et al., 2016) Adapted from Peterson and Artis (2014).

and progression of NAFLD through small intestinal bacterial overgrowth (SIBO) in conjunction with intestinal dysbiosis and increased permeability (Compare et al., 2012; Li et al., 2013; Miele et al., 2013; Vajro et al., 2013; Paolella et al., 2014). Recent experimental and clinical studies suggest that the gastrointestinal microbiome affects NAFLD pathogenesis through pathways that (i) facilitate metabolism and energy harvesting (Turnbaugh et al., 2006; Jiang et al., 2015), as described from high-fat fed mice models treated with high levels of pro-inflammatory cytokines that promote NAFLD development (Le Roy et al., 2013); (ii) dynamic interactions with the host's innate immune system where NAFLD is reported consequent to disrupted local immune cell functionality (Su et al., 2012).

\section{INTESTINAL MICROBIAL COMPOSITION AND NAFLD}

Widespread biodiversity exists in the microbial ecosystems of humans, particularly in the intestinal tract. However, despite the extensive variety of bacteria, four main phylum dominate in the intestines: Firmicutes, Bacteroidetes, Actinobacteria, and Proteobacteria (Mokhtari et al., 2017), with up to $90 \%$ of microbes estimated to belong to the Firmicutes and Bacteroidetes phyla (Eckburg et al., 2005). In both human and experimental model based studies, NAFLD has been associated with altered microbiome abundance, composition and dysbiosis (Wigg et al., 2001; Mouzaki et al., 2013; Zhu et al., 2013). Analyses have also correlated changes in microbiota composition with change in disease severity (Shavakhi et al., 2013; Eslamparast et al., 2014, 2015; Rahimlou et al., 2015; Yari et al., 2016).

In reviewing the literature, studies that have analyzed the intestinal microbial composition of NAFLD patients in comparison to healthy controls have reported identifying patterns or trends that can be associated with NAFLD development (Harris et al., 2012).

Studies that have profiled the intestinal microbiome of NAFLD patients report that specific configurational and compositional shifts are associated with intestinal epithelial 


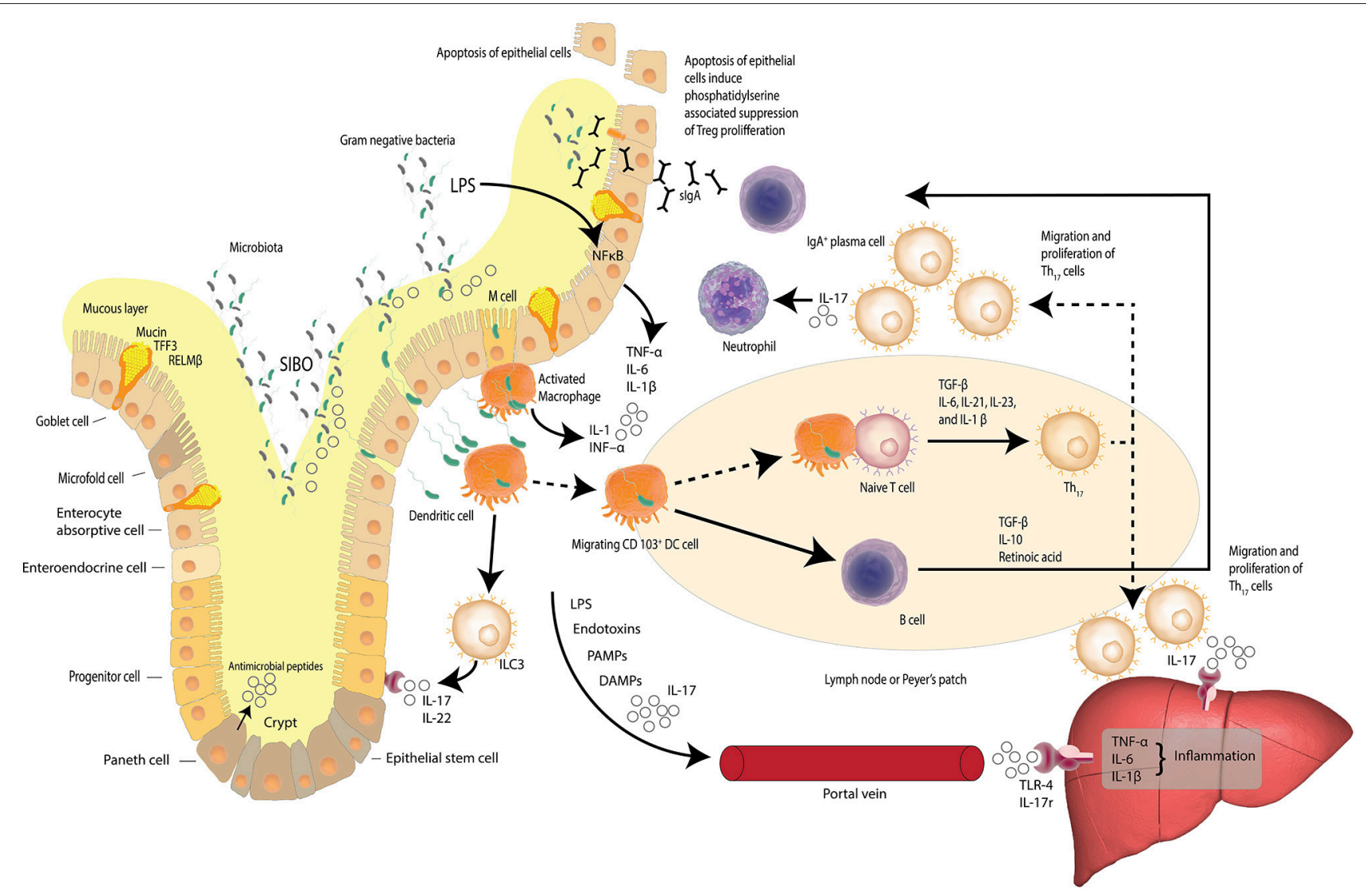

FIGURE 2 | Dysbiosis and the gut-liver axis. Increased intestinal permeability, small intestine bacterial overgrowth (SIBO) and elevated serum endotoxin like lipopolysaccharide (LPS) is found in patients with NAFLD. LPS, a hepatoxic component of gram-negative bacteria, is elevated in cases of SIBO. Increased intestinal permeability leads to increased bacterial translocation. Activated macrophages release inflammatory cytokines and dendritic cells stimulate the differentiation of naïve T cells to pro-inflammatory Th17 cells induced by TGF- $\beta$, IL-6, IL-23, and IL-1 $\beta$. Th17 cells secrete neutrophil-activating IL-17. Lymphoid Th17 also migrate to the liver where IL-17 stimulates monocytes, Kupffer cells, biliary epithelial cells, and stellate cells, to secrete pro-inflammatory cytokines and chemokines-inducing liver inflammation. LPS, bacterial endotoxins, IL-17, pathogen-associated molecular patterns (PAMPs) and damage-associated molecular patterns (DAMPs) may reach the liver via the portal vein and induce inflammation via Toll-like receptor 4 (TLR-4) and interleukin-17 (IL-17) receptors. The gut-liver axis is underlined by the fact that at least $70 \%$ of the liver's blood supply is delivered directly from the intestinal tract via the portal vein outflow of the intestine. (Zheng, 2013; Carding et al., 2015;

Robinson et al., 2015; Fukui, 2016; Kumar et al., 2016) Adapted from Peterson and Artis (2014).

cell dysbiosis and the elicitation of pro-inflammatory immune responses, central to NAFLD manifestation and progression (Kirpich et al., 2015). Specific bacteria have been associated with NAFLD phenotypes, serving as both antagonists and protagonists in NAFLD pathogenesis. Studies which profiled the intestinal microbiome of healthy controls and NAFLD patients across the spectrum, were able to identify families, genera and phyla that differed significantly in their abundance between the healthy controls and those with a NAFLD diagnosis. Whilst data is inconsistent regarding the association between the intestinal microbiome profile and NAFLD, patterns are emerging which highlight potential relationships between bacterial types and host health (Lau et al., 2015). A study comparing subjects diagnosed with NAFLD compared to lean adults, reported that gram negative bacteria were significantly enriched $(P=0.009)$ and gram positive bacteria were markedly decreased $(P=0.001)$ in the NAFLD cohort (Wang et al., 2016). Findings from similar studies confirm the strong relationship between the composition and configuration of the intestinal microbiome and fatty liver histologies, suggesting that adverse shifts in intestinal microbiome profiles are related to the development of NAFLD (Wang et al., 2016). In a prospective cross sectional study, a $20 \%$ increase in the Bacteroidetes phylum $(p=0.005)$ and a $24 \%$ decrease in Firmicutes $(p=0.002)$ was found in healthy controls in comparison to NAFLD patients (Wang et al., 2016). Interestingly, among the species belonging to the Firmicutes phylum, SCFA-producing bacteria were significantly decreased. Specific microbiome signatures of intestinal bacteria that are reported to be associated with significant reductions in butyric acid (Consolandi et al., 2015) may comprise a significant marker for the depletion of intestinal bacterial species that are important for the maintenance of intestinal barrier integrity and innate mucosal immunity equilibrium. However, currently it is difficult to identify which microbiome differences are causal and 
which are coincidental in the development of intestinal barrier dysbiosis and moreover NAFLD. Variations between studies, which profile microbiome composition of NAFLD patients, may in part be accounted for by different analytical techniques that have been employed. Furthermore, differences in study design, including anthropometric measures, markers used for NAFLD diagnosis as well as ultra-sonographic vs. biopsy NAFLD diagnosis adds additional discrepancies. As such Mouzaki et al. (2013), reported an inverse relationship between NASH diagnosis and the proportion of the phyla Bacteroidetes detected. These results were contrasted by Zhu et al (Zhu et al., 2013) who reported that NASH diagnosis was accompanied by higher levels of alcohol-producing bacteria and endogenous levels of ethanol, a result that was supported by Wong et al. (2013).

The intestinal microbial variations were also observed at the genus level, with data purporting to Ruminococcus and Roseburia genera being shown to be inconclusive. Whilst Zhu et al. (2013) and Raman et al. (2013) reported a nonsignificant decrease in the abundance of Ruminococcus in NAFLD patients compared to a group of healthy controls, whereas Del Chierico et al. (2017) and Jiang et al. (2015) found an increase in the genus' abundance in NAFLD patients.

Despite the various limitations, preliminary studies highlight and support the hypothesis that configurational shifts in the intestinal microbiome composition may contribute to the development and progression of NAFLD. Whilst further studies on a larger scale with accurate measures and controlled variables are warranted, the potential contribution of intestinal microorganisms and their metabolites are implicated in the pathophysiology of NAFLD. Studies that administer probiotics/prebiotics, which posit to encourage the intestinal microbiome to re-establish intestinal-mucosal macrophage crosstalk homeostasis that then translates to reducing the progression of NALFD are very much warranted.

\section{MECHANISMS LINKING THE INTESTINAL MICROBIOTA AND NAFLD}

Research identifies several mechanisms by which the intestinal microbiome cohort-arrangement can affect NAFLD pathogenesis and maintenance. Increased intestinal permeability (Jandhyala et al., 2015), small intestine bacterial overgrowth (SIBO) (Zhu et al., 2013) and elevated serum endotoxin like lipopolysaccharide (LPS) (Brun et al., 2007; Soares et al., 2010), have been reported by studies with NAFLD patients, with varying disease severity and staging (Elshaghabee et al., 2016). LPS, a component of gramnegative bacteria, is elevated in cases of bacterial overgrowth and increased intestinal permeability, yielding hepatoxic effects through the activation of TLR4 and initiation of a cascade of proinflammatory innate immune responses (Wigg et al., 2001; Soares et al., 2010).

Experimental and clinical data suggests that SIBO and a disturbed intestinal epithelial barrier are involved in NAFLD pathogenesis (Bode et al., 1987; Purohit et al., 2008; Wan et al., 2016). Furthermore, investigations have shown that the serum from NAFLD patients have elevated levels of LPS-binding protein, TLR-4, and TNF- $\alpha$ in hepatic tissue (Ruiz et al., 2007; Wan et al., 2016).

The gut microbiota also contributes to NAFLD pathogenesis through enriched numbers of ethanol-producing bacteria, primarily Escherichia coli (Small et al., 2013; Zhu et al., 2013; Jiang et al., 2015). The alcohol produced by these bacteria are reported to be involved in compromising intestinal barrier integrity which instigates inflammatory activity and ultimately hepatoxic events. Ethanol is a common and dominant metabolite of numerous resident intestinal microbes. As a product of hetero-lactic organisms, endogenously produced ethanol is implicated as a pro-inflammatory hepatoxic factor in NAFLD pathogenesis (Cope et al., 2000; Baker et al., 2010). Linked with increased concentrations of serum ethanol, enrichment in the presence of alcohol-producing intestinal bacteria, like E. coli, has been demonstrated to increase intestinal epithelial permeability (Aron-Wisnewsky et al., 2013; Jiang et al., 2015).

A prelude to the liver's averseness to excessive accumulation of FFAs, is intestinal dysbiosis. Dysbiosis is the concept that describes compositional alterations away from the conventional symbiotic intestinal microbiota that may be associated with pathology within the host and is visually described in Figure 2. Herein we further posit that compositional alterations in the intestinal microbiota adversely affect the intestinal epithelial barrier exacerbating epithelial cell dysbiosis. It has been shown that intestinal epithelial cell disruption can directly adversely affect intestinal resident macrophages and act as critical effector cells in the initiation of inflammation in the pathogenesis of metabolic diseases (Chawla et al., 2011). Disturbances of the TJP network which link the epithelial cells to form the intestinal barrier is a central regulatory mechanism, facilitating selective permeability across the intestinal mucosa and limiting bacterial translocation. Examining the duodenum of NAFLD patients and healthy adults has revealed that in comparison to NAFLD patients, (Jiang et al., 2015) the TJP network was significantly more intact in the duodenum of a healthy adult, with regular alignment and extensive abundance of the microvilli(Jiang et al., 2015). These observations are in stark contrast to the significantly wider gaps and disrupted TJPs reported in NAFLD patients, suggesting a loss of barrier integrity with a consequent increase in bacterial translocation through increased intestinal permeability (Briskey et al., 2016). Additional assessment of the serum biomarkers of the TJPs, including occludin proteins, which are structural components of the tight junctions, have been reported with significantly higher levels in the intestinal mucosa of healthy adults compared to NAFLD patients (Jiang et al., 2015). Measuring the serum levels of the proteins that comprise the structural backbone of the intestinal TJP network lends further supportive data to the hypothesis that intestinal mucosal permeability is greater in NAFLD patients than lean subjects or controls, suggesting intestinal epithelial dysbiosis is a causal factor in NAFLD pathogenesis.

With the establishment of the intestinal microbiota as a participant in the onset and maintenance of low-grade systemic inflammation, the probing of the intestinal microbiome as a 
potential therapeutic target for extra-intestinal inflammatory conditions, such as NAFLD begun.

The supposition that the intestinal microbiome could indirectly and adversely influence the physiological function of an end-organ such as the liver, by contributing proinflammatory activity in the intestinal mucosa, is a novel concept with biological plausibility. As an example, the uptake of Shigatoxin from the pathogen enterohemorrhagic $E$. coli by $\mathrm{M}$ cells and the underlying macrophages in the Peyer's patches is a critical step that teaches about bacterial translocation. This step has been correlated to the efficiency of the infection by the pathogen (Etienne-Mesmin et al., 2011). Numerous bacterial pathogens and their products cross the epithelial barrier though $\mathrm{M}$ cell junctions that are then subsequently captured by intestinal resident macrophages (Alisi et al., 2017). Moreover, the LPS components of bacteria are ligands of TLR4 that are expressed on various immune cells, including intestinal macrophages (Vijayan et al., 2017). TLR4 in the M1 configuration is a mediator of inflammation that may imply that increased LPS/TLR4 signaling could be a driving factor in the accelerated inflammation process in patients with NAFLD (Alisi et al., 2017). LPS also induces activation of genes on macrophages such as the early growth response gene 1 (EGR1) as well as the LPS-induced expression of TNF- $\alpha$, an action that is directly mediated through EGR1 and NF-kB (Xu et al., 2001). Therefore bacterial products that activate macrophages and other immune cells to produce proinflammatory mediators can trigger inflammation in an end organ such as the liver (Tateya et al., 2013). Signals released in response to microbial dangers are absorbed on a backgorund of increased and dysregulated intestinal barrier permeability. These signals are recognized by PRRs, including TLR-4 (Szabo et al., 2010; Wan et al., 2016), and when sensed by NLRP3, support the hypothesis that inflammasome-driven microflora are potential drivers of NAFLD onset (Wan et al., 2016).

Intestinal homeostasis is pivotal in optimal functionality of the innate immune system and hinges on macrophages eliminating pathogenic bacteria and their particles (Vitetta, 2016). Activated macrophages play a dual role within the innate immune system (Sansonetti, 2002; Vitetta, 2016). Firstly, they help to elicit appropriate immune responses to detected microbial proteins by facilitating the presentation of antigens to T lymphocytes (Sansonetti, 2002; Vitetta, 2016). Secondly, activated macrophages serve as a secretory source for an array of cytokines that regulate the activation of $\mathrm{T}$ cell lymphocytes, including IL-1, INF- $\alpha$, and cytotoxic proteins (Sansonetti, 2002; Vitetta, 2016). The overall action of the macrophage within the immune responses rely on their ability to neutralize exogenous antigens, cellular debris, insoluble particles, and activated clotting factors via phagocytic activity (Tacke, 2017).

\section{REPRISE}

NAFLD's pathway to pathogenicity is characterized by the presence of ectopic fat within hepatocytes that results from an imbalance in the levels of lipogenesis and lipolysis (Machado and Cortez-Pinto, 2014). Triglycerides are synthesized from FFAs that are reported to accumulate in the liver. Therefore, it is envisaged that the concentration of FFAs function as a regulator of lipogenesis in the liver.

Previous studies have associated bacterial phyla, families, or even single genera with obese or lean phenotypes, with an increased lactobacilli count and decreased Bacteroidetes presence associated with leanness (Armougom et al., 2009). In support of this, an increased abundance of genus' of the Bifidobacterium animalis or Lactobacillus species were associated with weight management and a healthy body weight (Million et al., 2012). Lactobacillus reuteri has specifically been identified as being associated with an obese phenotype (Million et al., 2012). Assessing intestinal microorganisms for possible correlations with NAFLD is a biologically plausible next step in progressing an understanding of how much influence the intestinal microbiome as a metabolic and immunological organ may have on the development and progression of NAFLD.

Methodological and technological difficulties have largely prevented robust and definitive data from studies that specifically assess the intestinal microbiota of adults with NAFLD and the health of the intestinal mucosal barrier as well as a need for further knowledge into what constitutes a healthy microbiota. When the mucosal barrier of the intestine, which also serves as the largest immune area of the intestinal immune system, is impaired and disrupted, the liver is exposed to intestinal-derived bacterial factors, which are potentially hepatoxic through the gut-liver axis.

A majority of the current literature details the involvement of the innate branch of the immune system in pro-inflammatory pathways leading to NAFLD pathogenesis and progression. However, the adaptive immune system is also implicated in NAFLD development (Ganz and Szabo, 2013; Sutti et al., 2016). Linking the innate and adaptive branches, natural killer (NK) cells are abundant in hepatic tissue and have been reported to influence the development of liver injury and fibrotic deposition that spark NASH materialization (Ganz and Szabo, 2013). Studies in both animal and human models have found a decrease in circulating $\mathrm{NK}$ cells in obese subjects compared to lean counterparts (Ganz and Szabo, 2013), with further exploratory investigations suggesting a reduction in their levels and thus activity may in turn increase the sensitivity of obese patients to develop progressive forms of NAFLD, including cirrhosis (Radaeva et al., 2006). A subset of NK cells, the natural killer T (NKT) cells, also known for their exhibition of both innate and adaptive immunity features, serve to regulate hepatic immune responses by secreting both Th1 and Th2 cytokines (Godfrey et al., 2000). Experimental and clinical data indicates that a depletion in NKT cells can lead to the chronic pro-inflammatory environment that can accompanies hepatic steatosis (Li et al., 2005; Ronchi and Falcone, 2008).

With no effective medication having yet been tested for managing or treating NAFLD and the only universally accepted treatment strategy being lifestyle modifications that focus of weight loss, novel therapeutic agents are being pursued in an endeavor to address the rise of NAFLD as one of the most common non-communicable liver disease world-wide (Volynets et al., 2012). 
Whilst lifestyle modification recommendations encourage weight loss, this approach requires significant commitment and efficiency decreases over the long term due to waning dedication. Intestinal microbial manipulation through the administration of probiotics presents as an attractive therapeutic adjunct. In an attempt to reduce intestinal epithelial inflammation, probiotics shift the intestinal microbial community toward beneficial bacterial communities such as the Parabacteroides, Prevotella, and Oscillibacter (Ohland and MacNaughton, 2010). These microbial communities are well known to produce antiinflammatory metabolites such as SCFAs (Schwiertz et al., 2010). SCFAs, such as butyrate, serve as important facilitators in the harvesting of energy and harnessing it for peripheral tissues and the intestinal epithelia (Elshaghabee et al., 2016). Oscillibacter and Parabacteroides are associated with T-cell differentiation by enhancing and maintaining the IL-10 producing Treg cells (Arpaia et al., 2013). Moreover, a probiotic formulation that attenuated hepatocellular carcinoma in a murine model offered further insight on how the intestinal microbiota influences the regulation of T-cell differentiation of mucosal immunity in the intestines and in turn, there is down-regulation of the level of pro-inflammatory cytokines ( $\mathrm{Li}$ et al., 2016). A recently completed murine study by our group reported attenuation of steatosis by $60 \%$ in a high fat diet model of NAFLD (Briskey et al., 2016). As such the administration of probiotics to attenuate the progression of NAFLD is both clinically plausible and very much warranted.

Despite increasing focus and attention directed at identifying the mechanisms by which NAFLD develops and progresses, ambiguity remains surrounding the driving factors and molecular pathways that result in NAFLD onset. Immunological mechanisms, including the collaboration of the consequences of innate immunity, adaptive immunity, and TLR receptor

\section{REFERENCES}

Abbas, A. K., Benoist, C., Bluestone, J. A., Campbell, D. J., Ghosh, S., Hori, S., et al. (2013). Regulatory T cells: recommendations to simplify the nomenclature. Nat. Immunol. 14, 307-308. doi: 10.1038/ni.2554

Adams, L. A., Lymp, J. F., St Sauver, J., Sanderson, S. O., Lindor, K. D., Feldstein, A., et al. (2005). The natural history of nonalcoholic fatty liver disease: a population-based cohort study. Gastroenterology 129, 113-121. doi: 10.1053/j.gastro.2005.04.014

Ai, T. L., Solomon, B. D., and Hsieh, C. S. (2014). T-cell selection and intestinal homeostasis. Immunol. Rev. 259, 60-74. doi: 10.1111/imr.12171

Alisi, A., Carpino, G., Oliveira, F. L., Panera, N., Nobili, V., and Gaudio, E. (2017). The role of tissue macrophage-mediated inflammation on nafld pathogenesis and its clinical implications. Mediators Inflamm. 2017:8162421. doi: $10.1155 / 2017 / 8162421$

Armougom, F., Henry, M., Vialettes, B., Raccah, D., and Raoult, D. (2009). Monitoring bacterial community of human gut microbiota reveals an increase in Lactobacillus in obese patients and methanogens in anorexic patients. PLoS ONE 4:e7125. doi: 10.1371/journal.pone.0007125

Aron-Wisnewsky, J., Gaborit, B., Dutour, A., and Clement, K. (2013). Gut microbiota and non-alcoholic fatty liver disease: new insights. Clin. Microbiol. Infect. 19, 338-348. doi: 10.1111/1469-0691.12140

Arpaia, N., Campbell, C., Fan, X., Dikiy, S., van der Veeken, J., deRoos, P., et al. (2013). Metabolites produced by commensal bacteria promote peripheral regulatory T-cell generation. Nature 504, 451-455. doi: 10.1038/nature12726 signaling dysfunction with the gut-liver axis are each posited to contribute to disease pathogenesis and maintenance.

This narrative review has highlighted the requisite for the completion of a systematic literature review detailing the association between innate immune responses triggered by intestinal epithelial inflammation and dysbiosis and the onset of extra-intestinal pathologies, such as NAFLD. The intestinal microbiome is a significant environmental factor in NAFLD pathogenesis, specifically through effects on intestinal barrier integrity. A review of the literature which explores the correlation between changes in intestinal integrity, intestinal permeability, and therefore endotoxin translocation will help elucidate the specific patterns or profiles of intestinal microorganisms that are of interest in NAFLD manifestation and therefore relevant for therapeutic target purposes. Furthermore, such a review may also define bacterial species that elicit a hepatoprotective effect on the microbiome and extra-intestinal inflammation.

\section{AUTHOR CONTRIBUTIONS}

ES and LV: Conception and design of commentary, review; ES, LV, MT, and TP: Read, amended and approved final version of manuscript.

\section{FUNDING}

LV has received National Institute of Complementary Medicine and National Health and Medical Research Council of Australia competitive funding and Industry support for research into biocompounds and probiotics. ES, LV, and MT participate in research on probiotics in Medlab Clinical's research laboratory facility in Sydney, Australia.
Baker, S. S., Baker, R. D., Liu, W., Nowak, N. J., and Zhu, L. (2010). Role of alcohol metabolism in non-alcoholic steatohepatitis. PLoS ONE 5:e9570. doi: 10.1371/journal.pone.0009570

Bieghs, V., and Trautwein, C. (2014). Innate immune signaling and gut-liver interactions in non-alcoholic fatty liver disease. Hepatobiliary Surg. Nutr. 3, 377-385. doi: 10.3978/j.issn.2304-3881.2014.12.04

Bode, C., Kugler, V., and Bode, J. C. (1987). Endotoxemia in patients with alcoholic and non-alcoholic cirrhosis and in subjects with no evidence of chronic liver disease following acute alcohol excess. J. Hepatol. 4, 8-14. doi: 10.1016/S0168-8278(87)80003-X

Boulangé, C. L., Neves, A. L., Chilloux, J., Nicholson, J. K., and Dumas, M.-E. (2016). Impact of the gut microbiota on inflammation, obesity, and metabolic disease. Genome Med. 8:42. doi: 10.1186/s13073-016-0303-2

Brandl, K., Kumar, V., and Eckmann, L. (2017). Gut-liver axis at the frontier of host-microbial interactions. Am. J. Physiol. Gastrointest. Liver physiol. 312, G413-g9. doi: 10.1152/ajpgi.00361.2016

Briskey, D., Heritage, M., Jaskowski, L. A., Peake, J., Gobe, G., Subramaniam, V. N., et al. (2016). Probiotics modify tight-junction proteins in an animal model of nonalcoholic fatty liver disease. Therap. Adv. Gastroenterol. 9, 463-472. doi: 10.1177/1756283X16645055

Brun, P., Castagliuolo, I., Di Leo, V., Buda, A., Pinzani, M., Palù, G., et al. (2007). Increased intestinal permeability in obese mice: new evidence in the pathogenesis of nonalcoholic steatohepatitis. Am. J. Physiol. Gastrointestinal Liver Physiol. 292, G518-G525. doi: 10.1152/ajpgi.00024. 2006 
Cani, P. D., Bibiloni, R., Knauf, C., Waget, A., Neyrinck, A. M., Delzenne, N. M., et al. (2008). Changes in gut microbiota control metabolic endotoxemiainduced inflammation in high-fat diet-induced obesity and diabetes in mice. Diabetes 57, 1470-1481. doi: 10.2337/db07-1403

Carding, S., Verbeke, K., Vipond, D. T., Corfe, B. M., and Owen, L. J. (2015). Dysbiosis of the gut microbiota in disease. Microb. Ecol. Health Dis. 26:26191. doi: 10.3402/mehd.v26.26191

Chawla, A., Nguyen, K. D., and Goh, Y. P. (2011). Macrophage-mediated inflammation in metabolic disease. Nat. Rev. Immunol. 11, 738-749. doi: $10.1038 /$ nri3071

Chiang, C. H., Huang, C. C., Chan, W. L., Chen, J. W., and Leu, H. B. (2010). The severity of non-alcoholic fatty liver disease correlates with high sensitivity C-reactive protein value and is independently associated with increased cardiovascular risk in healthy population. Clin. Biochem. 43, 1399-1404. doi: 10.1016/j.clinbiochem.2010.09.003

Compare, D., Coccoli, P., Rocco, A., Nardone, O. M., De Maria, S., Cartenì, M., et al. (2012). Gut-liver axis: the impact of gut microbiota on non alcoholic fatty liver disease. Nutr. Metab. Cardiovasc. Dis. 22, 471-476. doi: 10.1016/j.numecd.2012.02.007

Consolandi, C., Turroni, S., Emmi, G., Severgnini, M., Fiori, J., Peano, C., et al. (2015). Behcet's syndrome patients exhibit specific microbiome signature. Autoimmun. Rev. 14, 269-276. doi: 10.1016/j.autrev.2014.11.009

Cope, K., Risby, T., and Diehl, A. M. (2000). Increased gastrointestinal ethanol production in obese mice: implications for fatty liver disease pathogenesis. Gastroenterology 119, 1340-1347. doi: 10.1053/gast.2000.19267

Day, C. P., and James, O. F. (1998). Steatohepatitis: a tale of two "hits"? Gastroenterology 114, 842-845. doi: 10.1016/S0016-5085(98)70599-2

Del Chierico, F., Nobili, V., Vernocchi, P., Russo, A., Stefanis, C., Gnani, D., et al. (2017). Gut microbiota profiling of pediatric nonalcoholic fatty liver disease and obese patients unveiled by an integrated meta-omics-based approach. Hepatology 65, 451-464. doi: 10.1002/hep.28572

Dietrich, P., and Hellerbrand, C. (2014). Non-alcoholic fatty liver disease, obesity and the metabolic syndrome. Best Pract. Res. Clin. Gastroenterol. 28, 637-653. doi: 10.1016/j.bpg.2014.07.008

Dunn, W., Xu, R., Wingard, D. L., Rogers, C., Angulo, P., Younossi, Z. M., et al. (2008). Suspected nonalcoholic fatty liver disease and mortality risk in a population-based cohort study. Am. J. Gastroenterol. 103, 2263-2271. doi: 10.1111/j.1572-0241.2008.02034.X

Eckburg, P. B., Bik, E. M., Bernstein, C. N., Purdom, E., Dethlefsen, L., Sargent, M., et al. (2005). Diversity of the human intestinal microbial flora. Science 308, 1635-1638. doi: 10.1126/science.1110591

Elshaghabee, F. M., Bockelmann, W., Meske, D., de Vrese, M., Walte, H.G., Schrezenmeir, J., et al. (2016). Ethanol production by selected intestinal microorganisms and lactic acid bacteria growing under different nutritional conditions. Front. Microbiol. 7:47. doi: 10.3389/fmicb.2016.00047

Eslamparast, T., Eghtesad, S., Poustchi, H., and Hekmatdoost, A. (2015). Recent advances in dietary supplementation, in treating non-alcoholic fatty liver disease. World J. Hepatol. 7, 204-212. doi: 10.4254/wjh.v7.i2.204

Eslamparast, T., Poustchi, H., Zamani, F., Sharafkhah, M., Malekzadeh, R., and Hekmatdoost, A. (2014). Synbiotic supplementation in nonalcoholic fatty liver disease: a randomized, double-blind, placebo-controlled pilot study. Am. J. Clin. Nutr. 99, 535-542. doi: 10.3945/ajcn.113.068890

Etienne-Mesmin, L., Chassaing, B., Sauvanet, P., Denizot, J., Blanquet-Diot, S., Darfeuille-Michaud, A., et al. (2011). Interactions with $\mathrm{M}$ cells and macrophages as key steps in the pathogenesis of enterohemorrhagic Escherichia coli infections. PLoS ONE 6:e23594. doi: 10.1371/journal.pone.0023594

Farrell, G. C., van Rooyen, D., Gan, L., and Chitturi, S. (2012). NASH is an inflammatory disorder: pathogenic, prognostic and therapeutic implications. Gut Liver 6, 149-171. doi: 10.5009/gnl.2012.6.2.149

Fukui, H. (2016). Increased intestinal permeability and decreased barrier function: does it really influence the risk of inflammation? Inflamm. Intest. Dis. 1, 135-145. doi: 10.1159/000447252

Gaggini, M., Morelli, M., Buzzigoli, E., DeFronzo, R. A., Bugianesi, E., and Gastaldelli, A. (2013). Non-Alcoholic Fatty Liver Disease (NAFLD) and its connection with insulin resistance, dyslipidemia, atherosclerosis and coronary heart disease. Nutrients 5, 1544-1560. doi: 10.3390/nu5051544

Ganz, M., and Szabo, G. (2013). Immune and inflammatory pathways in NASH. Hepatol. Int. 7, 771-781. doi: 10.1007/s12072-013-9468-6
Glavan, T. W., Gaulke, C. A., Santos Rocha, C., Sankaran-Walters, S., Hirao, L. A., Raffatellu, M., et al. (2016). Gut immune dysfunction through impaired innate pattern recognition receptor expression and gut microbiota dysbiosis in chronic SIV infection. Mucosal Immunol. 9, 677-688. doi: 10.1038/mi.2015.92

Godfrey, D. I., Hammond, K. J., Poulton, L. D., Smyth, M. J., and Baxter, A. G. (2000). NKT cells: facts, functions and fallacies. Immunol. Today 21, 573-583. doi: 10.1016/S0167-5699(00)01735-7

Gottschalk, C., and Kurts, C. (2015). The debate about dendritic cells and macrophages in the kidney. Front. Immunol. 6:435. doi: 10.3389/fimmu.2015. 00435

Gregor, M. F., and Hotamisligil, G. S. (2011). Inflammatory mechanisms in obesity. Annu. Rev. Immunol. 29, 415-445. doi: 10.1146/annurev-immunol-031210-1 01322

Guilliams, M., Ginhoux, F., Jakubzick, C., Naik, S. H., Onai, N., Schraml, B. U., et al. (2014). Dendritic cells, monocytes and macrophages: a unified nomenclature based on ontogeny. Nat. Rev. Immunol. 14, 571-578. doi: 10.1038/nri3712

Hammerich, L., Heymann, F., and Tacke, F. (2011). Role of IL-17 and Th17 cells in liver diseases. Clin. Dev. Immunol. 2011:345803. doi: 10.1155/2011/345803

Harley, I. T., Stankiewicz, T. E., Giles, D. A., Softic, S., Flick, L. M., Cappelletti, M., et al. (2014). IL-17 signaling accelerates the progression of nonalcoholic fatty liver disease in mice. Hepatology 59, 1830-1839. doi: 10.1002/hep.26746

Harris, K., Kassis, A., Major, G., and Chou, C. J. (2012). Is the gut microbiota a new factor contributing to obesity and its metabolic disorders? J. Obes. 2012:879151. doi: 10.1155/2012/879151

Hume, D. A. (2008). Macrophages as APC and the dendritic cell myth. J. Immunol. 181, 5829-5835. doi: 10.4049/jimmunol.181.9.5829

Jandhyala, S. M., Talukdar, R., Subramanyam, C., Vuyyuru, H., Sasikala, M., and Nageshwar Reddy, D. (2015). Role of the normal gut microbiota. World J. Gastroenterol. 21, 8787-8803. doi: 10.3748/wjg.v21.i29.8787

Jiang, W., Wu, N., Wang, X., Chi, Y., Zhang, Y., Qiu, X., et al. (2015). Dysbiosis gut microbiota associated with inflammation and impaired mucosal immune function in intestine of humans with non-alcoholic fatty liver disease. Sci. Rep. 5:8096. doi: 10.1038/srep08096

Kayama, H., Ueda, Y., Sawa, Y., Jeon, S. G., Ma, J. S., Okumura, R., et al. (2012). Intestinal CX3C chemokine receptor 1(high) (CX3CR1(high)) myeloid cells prevent T-cell-dependent colitis. Proc. Natl. Acad. Sci. U.S.A. 109, 5010-5015. doi: 10.1073/pnas.1114931109

Kidd, P. (2003). Th1/Th2 balance: the hypothesis, its limitations, and implications for health and disease. Altern. Med. Rev. 8, 223-246.

Kinnebrew, M. A., and Pamer, E. G. (2012). Innate immune signaling in defense against intestinal microbes. Immunol. Rev. 245, 113-131. doi: 10.1111/j.1600-065X.2011.01081.x

Kirpich, I. A., Parajuli, D., and McClain, C. J. (2015). Microbiome in NAFLD and ALD. Clin. Liver Dis. 6, 55-58. doi: 10.1002/cld.494

Kumar, H., Kawai, T., and Akira, S. (2011). Pathogen recognition by the innate immune system. Int. Rev. Immunol. 30, 16-34. doi: 10.3109/08830185.2010.529976

Kumar, P., Monin, L., Castillo, P., Elsegeiny, W., Horne, W., Eddens, T., et al. (2016). Intestinal interleukin-17 receptor signaling mediates reciprocal control of the gut microbiota and autoimmune inflammation. Immunity 44, 659-671. doi: 10.1016/j.immuni.2016.02.007

Lau, E., Carvalho, D., and Freitas, P. (2015). Gut microbiota: association with NAFLD and metabolic disturbances. Biomed Res. Int. 2015:979515. doi: 10.1155/2015/979515

Le Roy, T., Llopis, M., Lepage, P., Bruneau, A., Rabot, S., Bevilacqua, C., et al. (2013). Intestinal microbiota determines development of non-alcoholic fatty liver disease in mice. Gut 62, 1787-1794. doi: 10.1136/gutjnl-2012-3 03816

Li, D. Y., Yang, M., Edwards, S., and Ye, S. Q. (2013). Nonalcoholic fatty liver disease: for better or worse, blame the gut microbiota? J. Parenteral Enteral Nutr. 37, 787-793. doi: 10.1177/0148607113481623

Li, J., Sung, C. Y., Lee, N., Ni, Y., Pihlajamäki, J., Panagiotou, G., et al. (2016). Probiotics modulated gut microbiota suppresses hepatocellular carcinoma growth in mice. Proc. Natl. Acad. Sci. U.S.A. 113, E1306-E1315. doi: 10.1073/pnas.1518189113

Li, Z., Soloski, M. J., and Diehl, A. M. (2005). Dietary factors alter hepatic innate immune system in mice with nonalcoholic fatty liver disease. Hepatology 42, 880-885. doi: 10.1002/hep.20826 
Littman, D. R., and Pamer, E. G. (2011). Role of the commensal microbiota in normal and pathogenic host immune responses. Cell Host Microbe 10, 311-323. doi: 10.1016/j.chom.2011.10.004

Lumeng, C. N., and Saltiel, A. R. (2011). Inflammatory links between obesity and metabolic disease. J. Clin. Invest. 121, 2111-2117. doi: 10.1172/JCI57132

Machado, M. V., and Cortez-Pinto, H. (2014). Non-alcoholic fatty liver disease: what the clinician needs to know. World J. Gastroenterol. 20, 12956-12980. doi: 10.3748 /wjg.v20.i36.12956

Mehal, W. Z. (2012). The gut-liver axis: a busy two-way street. Hepatology 55, 1647-1649. doi: 10.1002/hep.25704

Miele, L., Marrone, G., Lauritano, C., Cefalo, C., Gasbarrini, A., Day, C., et al. (2013). Gut-liver axis and microbiota in NAFLD: insight pathophysiology for novel therapeutic target. Curr. Pharm. Des. 19, 5314-5324. doi: 10.2174/1381612811319290011

Miele, L., Valenza, V., La Torre, G., Montalto, M., Cammarota, G., Ricci, R., et al. (2009). Increased intestinal permeability and tight junction alterations in nonalcoholic fatty liver disease. Hepatology 49, 1877-1887. doi: 10.1002/hep. 22848

Million, M., Maraninchi, M., Henry, M., Armougom, F., Richet, H., Carrieri, P., et al. (2012). Obesity-associated gut microbiota is enriched in Lactobacillus reuteri and depleted in Bifidobacterium animalis and Methanobrevibacter smithii. Int. J. Obes. 36, 817-825. doi: 10.1038/ijo.2011.153

Mokhtari, Z., Gibson, D. L., and Hekmatdoost, A. (2017). Nonalcoholic fatty liver disease, the gut microbiome, and diet. Adv. Nutr. 8, 240-252. doi: 10.3945/an.116.013151

Mouzaki, M., Comelli, E. M., Arendt, B. M., Bonengel, J., Fung, S. K., Fischer, S. E., et al. (2013). Intestinal microbiota in patients with nonalcoholic fatty liver disease. Hepatology 58, 120-127. doi: 10.1002/hep.26319

Nakahashi-Oda, C., Udayanga, K. G., Nakamura, Y., Nakazawa, Y., Totsuka, N., Miki, H., et al. (2016). Apoptotic epithelial cells control the abundance of Treg cells at barrier surfaces. Nat. Immunol. 17, 441-450. doi: 10.1038/ni.3345

Nicholson, J. K., Holmes, E., Kinross, J., Burcelin, R., Gibson, G., Jia, W., et al. (2012). Host-gut microbiota metabolic interactions. Science 336, 1262-1267. doi: $10.1126 /$ science. 1223813

Nielsen, J. S., Larsson, A., Ledet, T., Turina, M., Tønnesen, E., and Krog, J. (2012). Rough-form lipopolysaccharide increases apoptosis in human CD4(+) and CD8(+) T lymphocytes. Scand. J. Immunol. 75, 193-202. doi: 10.1111/j.1365-3083.2011.02613.x

Nobili, V., Giorgio, V., Liccardo, D., Bedogni, G., Morino, G., Alisi, A., et al. (2014). Vitamin D levels and liver histological alterations in children with nonalcoholic fatty liver disease. Eur. J. Endocrinol. 170, 547-553. doi: 10.1530/EJE-13-0609

Nolan, J. P. (2010). The role of intestinal endotoxin in liver injury: a long and evolving history. Hepatology 52, 1829-1835. doi: 10.1002/hep.23917

Norimatsu, M., Ono, T., Aoki, A., Ohishi, K., Takahashi, T., Watanabe, G., et al. (1995). Lipopolysaccharide-induced apoptosis in swine lymphocytes in vivo. Infect. Immun. 63, 1122-1126.

Ogino, H., Nakamura, K., Ihara, E., Akiho, H., and Takayanagi, R. (2011). CD4+CD25+ regulatory T cells suppress Th17-responses in an experimental colitis model. Dig. Dis. Sci. 56, 376-386. doi: 10.1007/s10620-010-1286-2

Ohland, C. L., and Macnaughton, W. K. (2010). Probiotic bacteria and intestinal epithelial barrier function. Am. J. Physiol. Gastrointest. Liver Physiol. 298, G807-G819. doi: 10.1152/ajpgi.00243.2009

Paolella, G., Mandato, C., Pierri, L., Poeta, M., Di Stasi, M., and Vajro, P. (2014). Gut-liver axis and probiotics: Their role in non-alcoholic fatty liver disease. World J. Gastroenterol. 20, 15518-15531. doi: 10.3748/wjg.v20.i42.15518

Paquissi, F. C. (2016). Immune imbalances in non-alcoholic fatty liver disease: from general biomarkers and neutrophils to interleukin-17 axis activation and new therapeutic targets. Front. Immunol. 7:490. doi: 10.3389/fimmu.2016.00490

Pedra, J. H., Cassel, S. L., and Sutterwala, F. S. (2009). Sensing pathogens and danger signals by the inflammasome. Curr. Opin. Immunol. 21, 10-16. doi: 10.1016/j.coi.2009.01.006

Peterson, L. W., and Artis, D. (2014). Intestinal epithelial cells: regulators of barrier function and immune homeostasis. Nat. Rev. Immunol. 14, 141-153. doi: $10.1038 /$ nri3608

Purohit, V., Bode, J. C., Bode, C., Brenner, D. A., Choudhry, M. A., Hamilton, F., et al. (2008). Alcohol, intestinal bacterial growth, intestinal permeability to endotoxin, and medical consequences: summary of a symposium. Alcohol 42, 349-361. doi: 10.1016/j.alcohol.2008.03.131

Radaeva, S., Sun, R., Jaruga, B., Nguyen, V. T., Tian, Z., and Gao, B. (2006). Natural killer cells ameliorate liver fibrosis by killing activated stellate cells in NKG2D-dependent and tumor necrosis factor-related apoptosis-inducing ligand-dependent manners. Gastroenterology 130, 435-452. doi: 10.1053/j.gastro.2005.10.055

Rahimlou, M., Ahmadnia, H., and Hekmatdoost, A. (2015). Dietary supplements and pediatric non-alcoholic fatty liver disease: present and the future. World J. Hepatol. 7, 2597-2602. doi: 10.4254/wjh.v7. i25.2597

Ramachandran, A., Madesh, M., and Balasubramanian, K. A. (2000). Apoptosis in the intestinal epithelium: its relevance in normal and pathophysiological conditions. J. Gastroenterol. Hepatol. 15, 109-120. doi: 10.1046/j.1440-1746.2000.02059.x

Raman, M., Ahmed, I., Gillevet, P. M., Probert, C. S., Ratcliffe, N. M., Smith, S., et al. (2013). Fecal microbiome and volatile organic compound metabolome in obese humans with nonalcoholic fatty liver disease. Clin. Gastroenterol. Hepatol. 11, 868.e1-3-875.e1-3. doi: 10.1016/j.cgh.2013. 02.015

Ringel, Y., Maharshak, N., Ringel-Kulka, T., Wolber, E. A., Sartor, R. B., and Carroll, I. M. (2015). High throughput sequencing reveals distinct microbial populations within the mucosal and luminal niches in healthy individuals. Gut Microbes 6, 173-181. doi: 10.1080/19490976.2015.1044711

Robinson, K., Deng, Z., Hou, Y., and Zhang, G. (2015). Regulation of the intestinal barrier function by host defense peptides. Front. Vet. Sci. 2:57. doi: $10.3389 /$ fvets.2015.00057

Ronchi, F., and Falcone, M. (2008). Immune regulation by invariant NKT cells in autoimmunity. Front. Biosci. 13, 4827-4837. doi: 10.2741/3042

Ruiz, A. G., Casafont, F., Crespo, J., Cayón, A., Mayorga, M., Estebanez, A., et al. (2007). Lipopolysaccharide-binding protein plasma levels and liver TNF-alpha gene expression in obese patients: evidence for the potential role of endotoxin in the pathogenesis of non-alcoholic steatohepatitis. Obes. Surg. 17, 1374-1380. doi: 10.1007/s11695-007-9243-7

Sansonetti, P. (2002). Host-pathogen interactions: the seduction of molecular cross talk. Gut 50 (Suppl. 3):Iii2-Iii8. doi: 10.1136/gut.50.suppl_3.iii2

Schwiertz, A., Taras, D., Schäfer, K., Beijer, S., Bos, N. A., Donus, C., et al. (2010). Microbiota and SCFA in lean and overweight healthy subjects. Obesity 18, 190-195. doi: 10.1038/oby.2009.167

Shavakhi, A., Minakari, M., Firouzian, H., Assali, R., Hekmatdoost, A., and Ferns, G. (2013). Effect of a probiotic and metformin on liver aminotransferases in non-alcoholic steatohepatitis: a double blind randomized clinical trial. Int. J. Prev. Med. 4, 531-537.

Small, C. L., Reid-Yu, S. A., McPhee, J. B., and Coombes, B. K. (2013). Persistent infection with Crohn's disease-associated adherent-invasive Escherichia coli leads to chronic inflammation and intestinal fibrosis. Nat. Commun. 4:1957. doi: 10.1038/ncomms2957

Smith, P. M., Howitt, M. R., Panikov, N., Michaud, M., Gallini, C. A., Bohlooly, Y. M., et al. (2013). The microbial metabolites, short-chain fatty acids, regulate colonic Treg cell homeostasis. Science 341, 569-573. doi: $10.1126 /$ science. 1241165

Soares, J. B., Pimentel-Nunes, P., Roncon-Albuquerque, R., and Leite-Moreira, A. (2010). The role of lipopolysaccharide/toll-like receptor 4 signaling in chronic liver diseases. Hepatol. Int. 4, 659-672. doi: 10.1007/s12072-010-9219-x

Starley, B. Q., Calcagno, C. J., and Harrison, S. A. (2010). Nonalcoholic fatty liver disease and hepatocellular carcinoma: a weighty connection. Hepatology 51, 1820-1832. doi: 10.1002/hep.23594

Su, L., Wang, J. H., Cong, X., Wang, L. H., Liu, F., Xie, X. W., et al. (2012). Intestinal immune barrier integrity in rats with nonalcoholic hepatic steatosis and steatohepatitis. Chin. Med. J. 125, 306-311.

Sutti, S., Bruzzi, S., and Albano, E. (2016). The role of immune mechanisms in alcoholic and nonalcoholic steatohepatitis: a 2015 update. Expert Rev. Gastroenterol. Hepatol. 10, 243-253. doi: 10.1586/17474124.2016.1111758

Szabo, G., Bala, S., Petrasek, J., and Gattu, A. (2010). Gut-liver axis and sensing microbes. Dig. Dis. 28, 737-744. doi: 10.1159/000324281

Tacke, F. (2017). Targeting hepatic macrophages to treat liver diseases. J. Hepatol. 66, 1300-1312. doi: 10.1016/j.jhep.2017.02.026 
Takeuchi, O., and Akira, S. (2010). Pattern recognition receptors and inflammation. Cell 140, 805-820. doi: 10.1016/j.cell.2010.01.022

Targher, G., Bertolini, L., Padovani, R., Rodella, S., Tessari, R., Zenari, L., et al. (2007). Prevalence of nonalcoholic fatty liver disease and its association with cardiovascular disease among type 2 diabetic patients. Diabetes Care 30, 1212-1218. doi: 10.2337/dc06-2247

Targher, G., Bertolini, L., Padovani, R., Rodella, S., Zoppini, G., Pichiri, I., et al. (2010). Prevalence of non-alcoholic fatty liver disease and its association with cardiovascular disease in patients with type 1 diabetes. J. Hepatol. 53, 713-718. doi: 10.1016/j.jhep.2010.04.030

Tateya, S., Kim, F., and Tamori, Y. (2013). Recent advances in obesityinduced inflammation and insulin resistance. Front. Endocrinol. 4:93. doi: $10.3389 /$ fendo. 2013.00093

Tilg, H., and Moschen, A. R. (2010). Evolution of inflammation in nonalcoholic fatty liver disease: the multiple parallel hits hypothesis. Hepatology 52, 1836-1846. doi: 10.1002/hep.24001

Turnbaugh, P. J., Ley, R. E., Mahowald, M. A., Magrini, V., Mardis, E. R., and Gordon, J. I. (2006). An obesity-associated gut microbiome with increased capacity for energy harvest. Nature 444, 1027-1031. doi: 10.1038/nature05414

Vajro, P., Paolella, G., and Fasano, A. (2013). Microbiota and gut-liver axis: their influences on obesity and obesity-related liver disease. J. Pediatr. Gastroenterol. Nutr. 56, 461-468. doi: 10.1097/MPG.0b013e318284abb5

Vijayan, V., Srinu, T., Karnati, S., Garikapati, V., Linke, M., Kamalyan, L., et al. (2017). A new immunomodulatory role for peroxisomes in macrophages activated by the TLR4 ligand lipopolysaccharide. J. Immunol. 198, 2414-2425. doi: 10.4049/jimmunol.1601596

Vitetta, L. (2016). Lactobacillus acidophilus restores functionality in uremic macrophages: plausible or lacking evidence? Dig. Dis. Sci. 61, 1417-1419. doi: 10.1007/s10620-016-4084-7

Vitetta, L., Coulson, S., Linnane, A. W., and Butt, H. (2013). The gastrointestinal microbiome and musculoskeletal diseases: a beneficial role for probiotics and prebiotics. Pathogens 2, 606-626. doi: 10.3390/pathogens2040606

Volynets, V., Küper, M. A., Strahl, S., Maier, I. B., Spruss, A., Wagnerberger, S., et al. (2012). Nutrition, intestinal permeability, and blood ethanol levels are altered in patients with Nonalcoholic Fatty Liver Disease (NAFLD). Dig. Dis. Sci. 57, 1932-1941. doi: 10.1007/s10620-012-2112-9

Wahl, S. M. (2007). Transforming growth factor-beta: innately bipolar. Curr. Opin. Immunol. 19, 55-62. doi: 10.1016/j.coi.2006.11.008

Wan, X., Xu, C., Yu, C., and Li, Y. (2016). Role of NLRP3 inflammasome in the progression of NAFLD to NASH. Can. J. Gastroenterol. Hepatol. 2016:6489012. doi: $10.1155 / 2016 / 6489012$

Wang, B., Jiang, X., Cao, M., Ge, J., Bao, Q., Tang, L., et al. (2016). Altered fecal microbiota correlates with liver biochemistry in nonobese patients with non-alcoholic fatty liver disease. Sci. Rep. 6:32002. doi: 10.1038/srep32002

Welsh, J. A., Karpen, S., and Vos, M. B. (2013). Increasing prevalence of nonalcoholic fatty liver disease among United States adolescents, 1988-1994 to 2007-2010. J. Pediatr. 162, 496.e1-500.e1. doi: 10.1016/j.jpeds.2012.08.043
Wieland, A., Frank, D. N., Harnke, B., and Bambha, K. (2015). Systematic review: microbial dysbiosis and nonalcoholic fatty liver disease. Aliment. Pharmacol. Ther. 42, 1051-1063. doi: 10.1111/apt.13376

Wigg, A. J., Roberts-Thomson, I. C., Dymock, R. B., McCarthy, P. J., Grose, R. H., and Cummins, A. G. (2001). The role of small intestinal bacterial overgrowth, intestinal permeability, endotoxaemia, and tumour necrosis factor alpha in the pathogenesis of non-alcoholic steatohepatitis. Gut 48, 206-211. doi: 10.1136/gut.48.2.206

Williams, C. D., Stengel, J., Asike, M. I., Torres, D. M., Shaw, J., Contreras, M., et al. (2011). Prevalence of nonalcoholic fatty liver disease and nonalcoholic steatohepatitis among a largely middle-aged population utilizing ultrasound and liver biopsy: a prospective study. Gastroenterology 140, 124-131. doi: 10.1053/j.gastro.2010.09.038

Wong, V. W., Tse, C., Lam, T. T., Wong, G. L., Chim, A. M., Chu, W. C., et al. (2013). Molecular characterization of the fecal microbiota in patients with nonalcoholic steatohepatitis - a longitudinal study. PLOS ONE 8:e62885. doi: 10.1371/journal.pone.0062885

Xu, Z., Dziarski, R., Wang, Q., Swartz, K., Sakamoto, K. M., and Gupta, D. (2001). Bacterial peptidoglycan-induced tnf-alpha transcription is mediated through the transcription factors Egr-1, Elk-1, and NF-kappaB. J. Immunol. 167, 6975-6982. doi: 10.4049/jimmunol.167.12.6975

Yari, Z., Rahimlou, M., Eslamparast, T., Ebrahimi-Daryani, N., Poustchi, H., and Hekmatdoost, A. (2016). Flaxseed supplementation in non-alcoholic fatty liver disease: a pilot randomized, open labeled, controlled study. Int. J. Food Sci. Nutr. 67, 461-469. doi: 10.3109/09637486.2016.1161011

Younossi, Z. M., Koenig, A. B., Abdelatif, D., Fazel, Y., Henry, L., and Wymer, M. (2016). Global epidemiology of nonalcoholic fatty liver disease-Meta-analytic assessment of prevalence, incidence, and outcomes. Hepatology 64, 73-84. doi: 10.1002/hep.28431

Zheng, S. G. (2013). Regulatory T cells vs Th17: differentiation of Th17 versus Treg, are the mutually exclusive? Am. J. Clin. Exp. Immunol. 2, 94-106.

Zhu, L., Baker, S. S., Gill, C., Liu, W., Alkhouri, R., Baker, R. D., et al. (2013) Characterization of gut microbiomes in nonalcoholic steatohepatitis (NASH) patients: a connection between endogenous alcohol and NASH. Hepatology 57, 601-609. doi: 10.1002/hep.26093

Conflict of Interest Statement: The authors declare that the research was conducted in the absence of any commercial or financial relationships that could be construed as a potential conflict of interest

Copyright (c) 2018 Saltzman, Palacios, Thomsen and Vitetta. This is an open-access article distributed under the terms of the Creative Commons Attribution License (CC $B Y)$. The use, distribution or reproduction in other forums is permitted, provided the original author(s) and the copyright owner are credited and that the original publication in this journal is cited, in accordance with accepted academic practice. No use, distribution or reproduction is permitted which does not comply with these terms. 